\title{
Finite Element Analysis on Heat Generation of Multiplate Wet Clutch Jin-Gang LIU ${ }^{1}$, Tian-Wei ZUO' ${ }^{1, a,{ }^{*}}$, Feng-Wu SHAN ${ }^{2}$, Jian-Wen CHEN ${ }^{3}$ \\ ${ }^{1}$ School of Mechanical Engineering, Xiangtan University, 411105 Xiangtan, China \\ 2Jiangling Motors New Energy Vehicles Co. Ltd, 330013 Nanchang, China \\ 3Jianglu Electromechanical Science \& Technology Co. Ltd. 411105 Xiangtan, China \\ aTian-Wei ZUO: 920132493@qq.com
}

Keywords: Wet clutch, Simulated calculation, Temperature field, Stress field.

\begin{abstract}
According to wet clutch friction temperature is too high, excessive thermal stress, and other issues during the engagement. It adopted ANSYS workbench software for finite element analysis and introduced direct coupling method to complete simulated calculation and analysed of the temperature field and stress field under two different conditions by the steel discs of the wet clutch. The calculation took into transfer situation of the frictional heat between the friction disc and the pairing steel discs with a consideration of a variety of displacement constraints. Simulation result showed that the temperature of each point on the friction pair increased as the radial distance away from the clutch shaft. Reducing the engagement time is beneficial to reduce the engagement temperature, but it will increase the impact force, affect the ride comfort, increase the engagement time will increase the engagement temperature. The investigation results will lay a foundation for further thermal manage and structure optimization of multiplate wet clutch.
\end{abstract}

\section{Introduction}

The wet multi-disk clutch is an essential constituent of Automobile automatic transmission and has advantages of good thermal conductivity, large transmission torque and easy automatic control. During clutch working, friction make the friction surface temperature increase rapidly, temperature gradient and thermal stress increase and the thermal elastic instability is easy to be produced under the local high temperature. As a consequence, these affects lead to the deformation of the friction element and even material loss and finally do an early damage on clutch. Therefore, the focuses of the study of wet multi-disc clutch are the temperature field, the stress field and the thermal-mechanical coupling[1,2]. On the basis of 3-D finite element model, by considering about contact, rotation, pressure and coupling conditions, and then calculate the temperature field and stress field by the direct coupling method of ANSYS workbench, so the distribution of temperature field and stress field of steel sheet under three conditions are compared.

\section{The Thermal-mechanical Coupling Stress Field}

\section{The Friction Heat Generating Principle}

There is a certain degree of surface roughness on the dual steel plate and friction plate of clutch. Friction generates heat because when the friction plate meets the dual steel plate, frictional contact and sliding friction are formed on the micro convex body of the surface and the dual steel sheet and the friction plate will generate heat due to friction[3].

\section{Thermal-mechanical Coupling Strain}

According to the theory of the thermal elasticity, the object expands when it is heated. When the object is heated inhomogeneously, it expands inhomogeneously and so the linear strain is produced. Suppose the linear expanding coefficients is $\alpha$, when the temperature is up to $\Delta T$, the thermal strain caused by expansion is[4]: 


$$
\varepsilon_{T}=\alpha \Delta T
$$

While the wet clutch is in engaging process, the steel sheet makes the thermal strain because of increasing temperature and the mechanical elastic strain, ${ }^{\varepsilon_{j}}$ is formed under the action of mechanical external force. According to the linear theory of stress, the stress and strain caused by mechanical external forces can be superimposed with the stress and strain caused by temperature. The total strain of these interaction is[5]

$$
\varepsilon=\varepsilon_{j}+\varepsilon_{T}
$$

The relationship between the stress vector, $\boldsymbol{\sigma}$ and the strain, $\varepsilon$ :

$$
\boldsymbol{\sigma}=\mathbf{D} \varepsilon
$$

In the formula, $\mathbf{D}$ is the material elastic matrix.

Add the formula (2) into the formula(3), the unit stress of friction steel sheet is:

$$
\boldsymbol{\sigma}=\mathbf{D}\left(\varepsilon_{j}+\varepsilon_{T}\right)
$$

The equilibrium relationship between the contact pressure $\mathbf{p}(x, y, z)$ on the engagements and the unit stress $\boldsymbol{\sigma}$ is:

$$
\int_{V} \mathbf{B} \boldsymbol{\sigma} d V=\mathbf{p}(x, y, z)
$$

In the formula, $\mathbf{B}$ is the transformation matrix between element strain and nodal displacement, $V$ is the volume of steel sheet.

From (2) to (5), we can calculate the stress field distribution after the mechanical and thermal elastic stress superimpose when friction plate and steel sheet are in engagement process. And the contact between the friction plate and the steel sheet is elastic contact and there is no mutual penetration between them. Then the iterated algorithm can be taken to check the contact, if the contact appears, it is supposed to be restrained on contact and produced the effort on node immediately[6].

\section{Calculation Process of Temperature Field and Stress Field}

\section{Calculation Hypotheses}

When temperature field of wet clutch friction pair and stress field are in finite element analysis, in order to have study on the temperature field and stress field distribution of dual steel sheet, hypothesis of friction plate and dual steel sheet are necessary[7].

(1) The engagement process of dual steel sheet and friction plate is short, therefore, the heat that is taken away by cooling and radiation can be ignored.

(2) The heat generated by the friction is completely absorbed by the dual steel sheet and the friction plate.

(3) The wear of dual steel sheet and friction plate in the engagement process is neglected and the physical properties of the dual steel sheet and the material of the friction plate are not changed with the temperature[8].

\section{Friction Pair Model}

The wet multi-disc clutch is composed by separated arrangement of multiple friction plates and steel sheets and heat production of friction pair is a typical nonlinear coupling problem. Its computation is enormous and the load and the structure of the friction pair are symmetrical, so half thickness of the friction plate and the steel sheet can be taken to be analyzed. Half of the friction plate is provided with an oil grove structure, however, this can bring great difficulties to the mesh generation and 
finite element nonlinear calculation. According to the literature[9,10], the ignorance of the oil groove has no affect to the accuracy of the temperature field of dual steel sheet. Therefore, to simplify the structure of the friction pairs and ignore the oil groove structure of friction plate, then 3-D calculation mode, Figure.1.can be established.

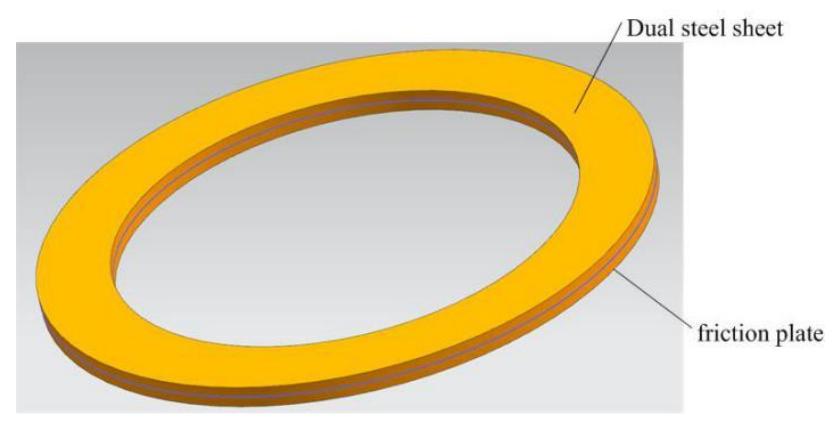

Figure 1. 3-D friction pair model

\section{Calculation Conditions}

In this model, inner diameters of friction pair are $85 \mathrm{~mm}$ and $112 \mathrm{~mm}$, friction linings are paper-based friction material. The experiment measures that the friction coefficient is 0.15 and friction pair material properties are shown in table 1.

Table 1. Material property parameter

\begin{tabular}{|l|l|l|}
\hline & Friction lining & Steel sheet \\
\hline Density $\left(\mathrm{kg} / \mathrm{m}^{3}\right)$ & 833 & 7850 \\
\hline Elastic modulus $(\mathrm{GPa})$ & 0.588 & 206 \\
\hline Poisson ratio & 0.12 & 0.30 \\
\hline mass heat capacity $(\mathrm{J} /(\mathrm{kg} * \mathrm{~K}))$ & 1740 & 490 \\
\hline Thermal conductivity $\left(\mathrm{W} /\left(\mathrm{m}^{*} \mathrm{~K}\right)\right)$ & 0.24 & 53 \\
\hline Thermal expansion coefficient $\left(10^{-5} \mathrm{~K}^{-1}\right)$ & 0.17 & 1.06 \\
\hline
\end{tabular}

In combination with the wet clutch engagement conditions, the relationship between the rotational speed and pressure of the clutch at different engagement time is obtained, as shown in Figure 2. and Figure 3.

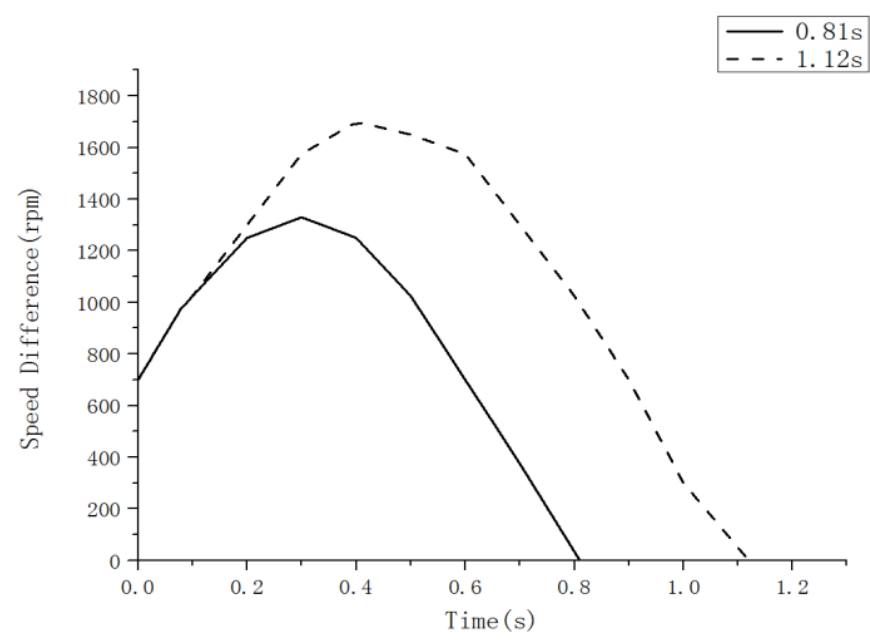

Figure 2. Speed difference curve of engagement process 


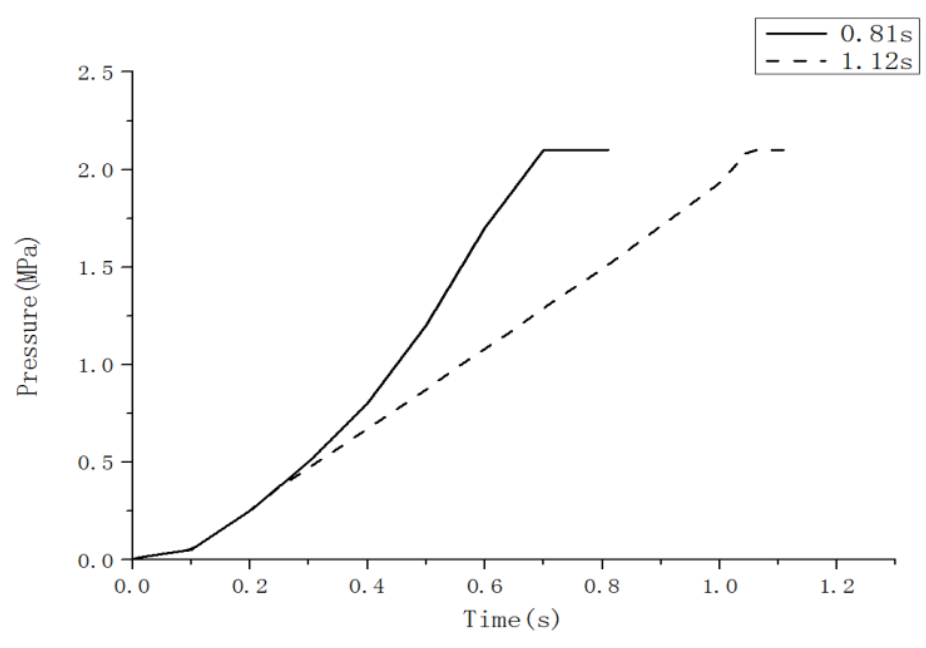

Figure 3. Pressure change curve of engagement process

A fixed constraint is applied to the symmetric surface of the friction plate, and the pressure is applied to the symmetric surface of the steel sheet, and the rotation displacement is applied to the inner ring surface of the steel sheet, to simulate the relative rotation.

\section{Results and Discussions}

As shown in Figure 4 is the dual sheet steel in the engagement time for stress distribution of $0.81 \mathrm{~s}$. The stress distribution of the whole engagement is relatively uniform, the maximum stress is 105.12MPa. As shown in Figure 5 is the dual sheet steel in the engagement time for stress distribution of $1.12 \mathrm{~s}$, the maximum stress is $190.59 \mathrm{MPa}$.From the two figure, we can see that the shorter the engagement process is, the greater the stress is, and the stress is mainly concentrated in the edge part. And the short time of the engagement is easy to have a larger impact, in the edge of the steel sheet will produce the distribution of wave shape.

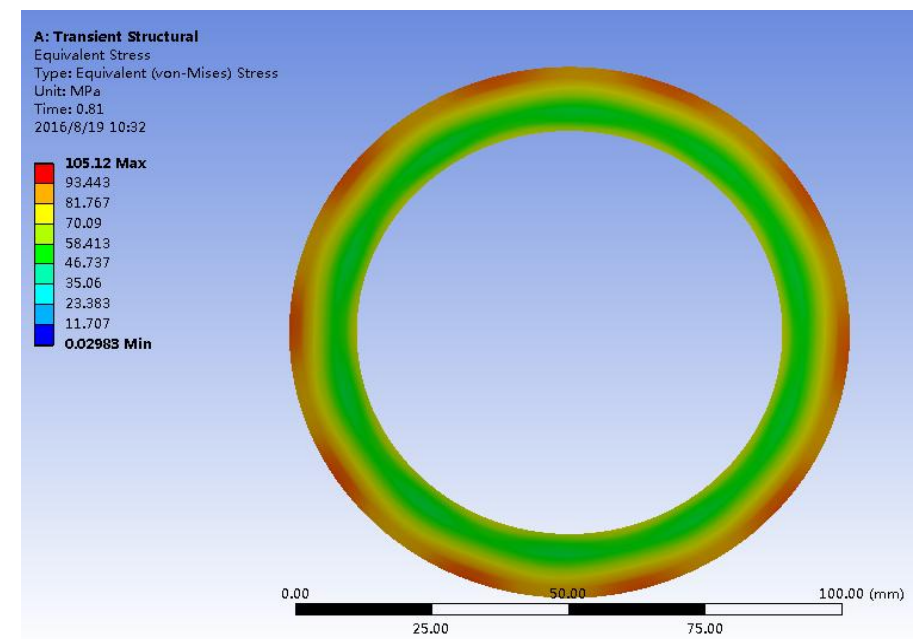

Figure 4.Stress distribution of dual steel sheets in $0.81 \mathrm{~s}$ 


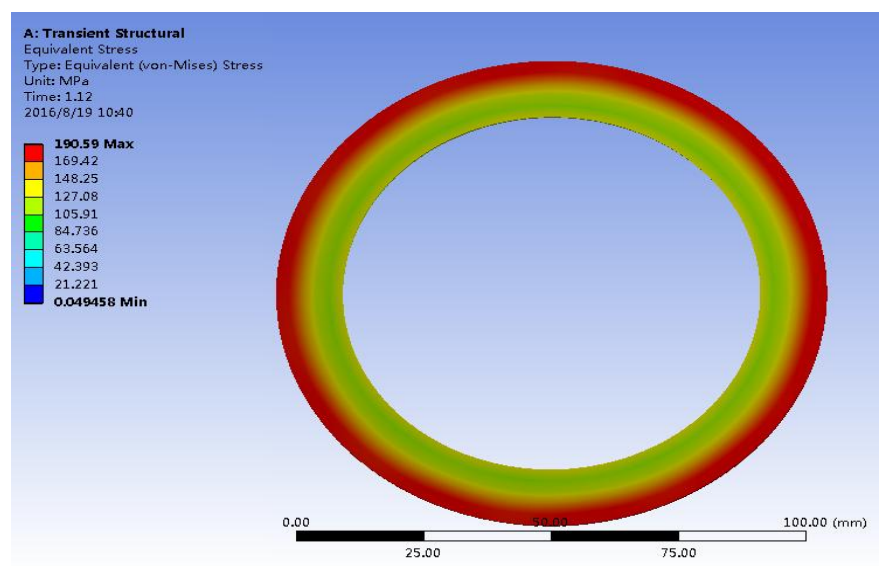

Figure 5.Stress distribution of dual steel sheets in $1.12 \mathrm{~s}$

The distribution of stress along the radius of the dual steel sheet is shown in figure 6, The higher the engagement time is, the higher the maximum stress and the stress gradient are, the distribution is not even. Along the radius direction, the stress first decreases, and then gradually increases at $44 \mathrm{~mm}$ to $45 \mathrm{~mm}$.

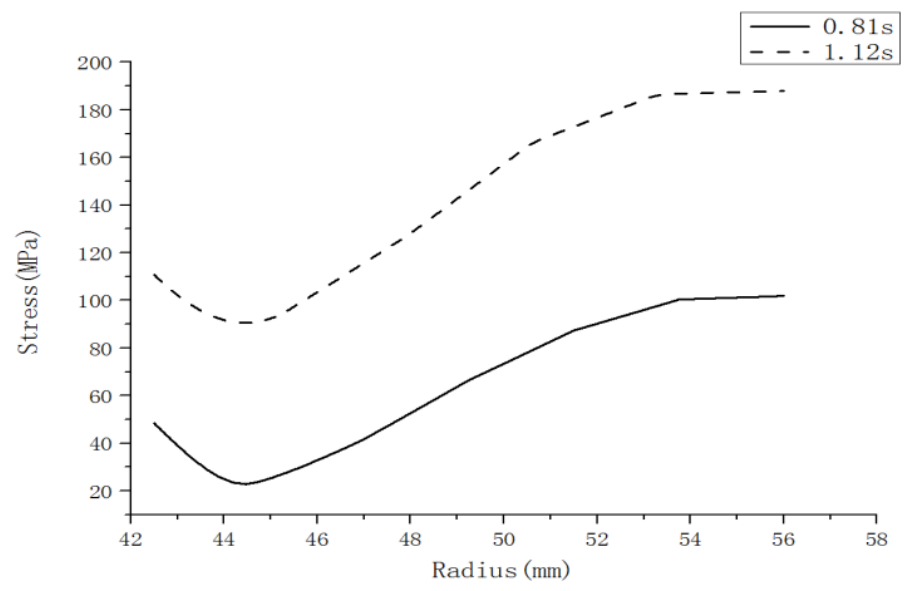

Figure 6.Distribution of stress along radius of dual steel sheet

Figure 7. shows when the engagement time is $0.81 \mathrm{~s}$, the engagement highest temperature is $116.17^{\circ} \mathrm{C}$.Figure 8 . shows the engagement time is $1.12 \mathrm{~s}$, the engagement highest temperature is $170.42^{\circ} \mathrm{C}$.As can be seen from the diagram, the longer the engagement time is, the higher the heat is, the higher the temperature is, the more heat is concentrated on the edge.

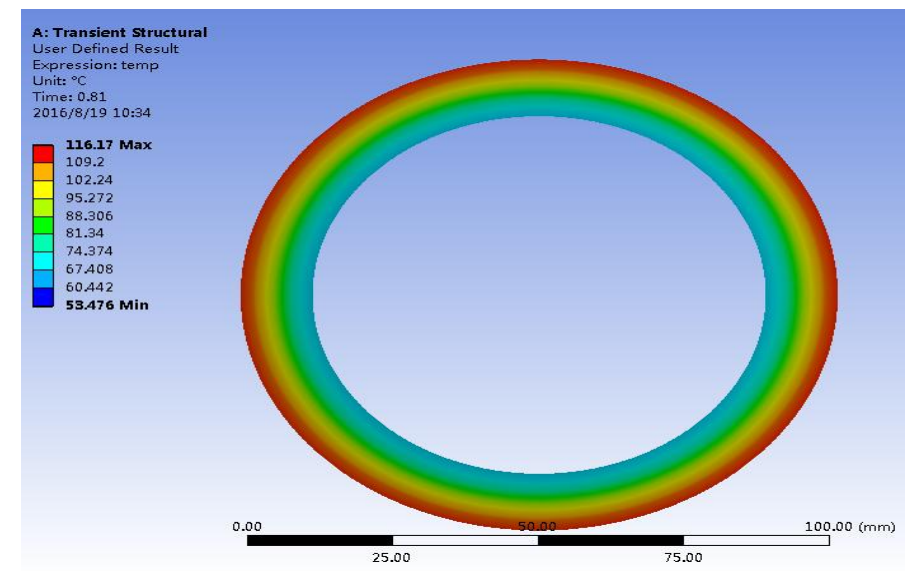

Figure 7.Temperature distribution of dual steel sheets in $0.81 \mathrm{~s}$ 


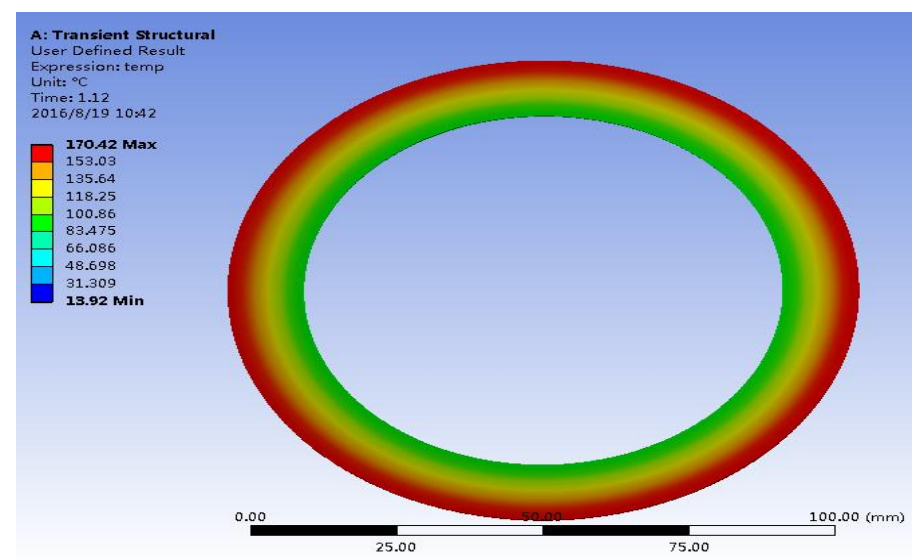

Figure 8.Temperature distribution of dual steel sheets in $1.12 \mathrm{~s}$

The temperature along the radius of the dual steel sheet is shown in Figure 9, which shows that the longer the engagement time is, the greater the temperature gradient is, and the increase of the temperature inhomogeneity.

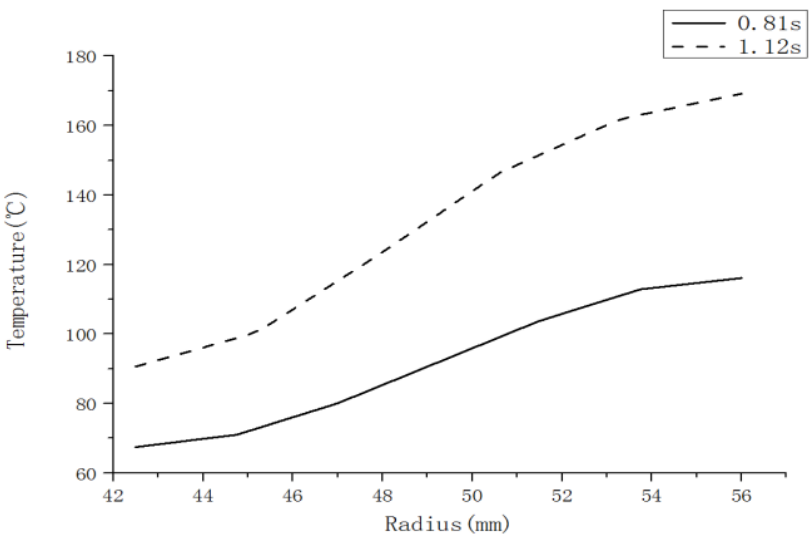

Figure 9.Distribution of temperature along radius of dual steel sheet

Figure 10 and Figure 11. shows the variation of the deformation along the axis of the engagement. Engagement surface showing a negative direction of $\mathrm{Z}$ axis of deformation. Figure 10 shows the engagement time is $0.81 \mathrm{~s}$, the maximum axial deformation of engagement surface is $0.0090219 \mathrm{~mm}$. Figure 11 shows the engagement time is $1.12 \mathrm{~s}$, the maximum axial deformation of engagement surface is $0.0079687 \mathrm{~mm}$. From the left can see the edge wave shape deformation, and stress distribution to fit, indicates that the engagement within a short period of time will have a greater impact, resulting in greater deformation.

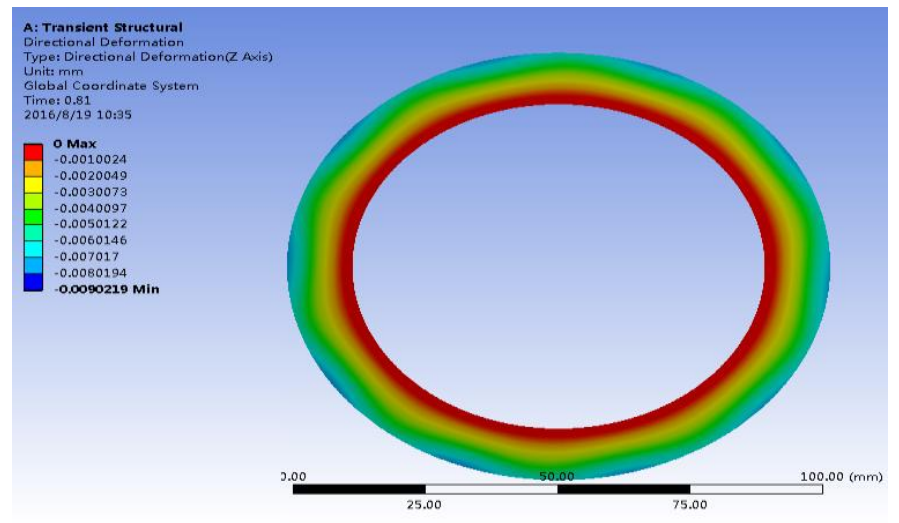

Figure 10.The distribution of axial deformation of dual steel sheet in $0.81 \mathrm{~s}$ 


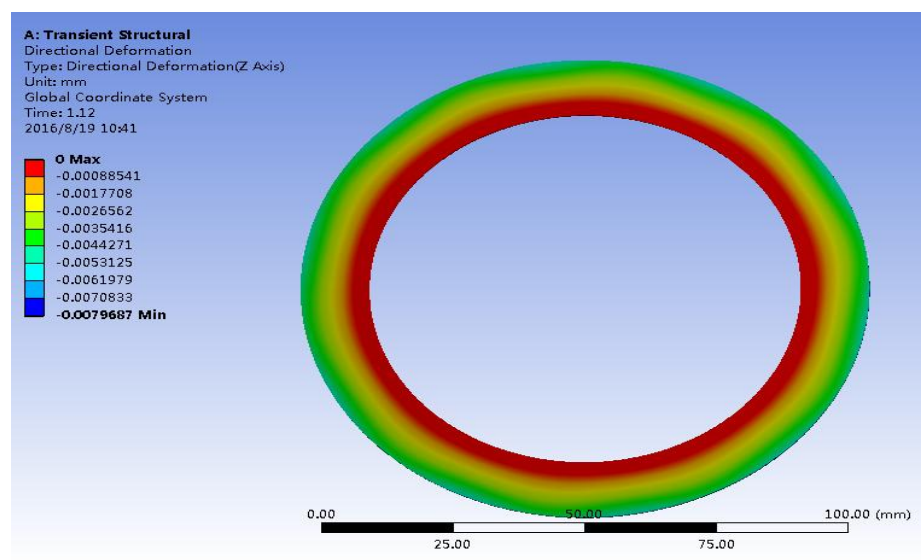

Figure 11.The distribution of axial deformation of dual steel sheet in $1.12 \mathrm{~s}$

Dual steel in axial direction along the radius direction deformation distribution as shown in Figure 12. It can be known that the deformation is gradually increased along the radius. And it can be clearly seen that the deformation of $0.81 \mathrm{~s}$ engagement time is larger, and the deformation gradient is greater. It shows that the longer engagement time is beneficial to reduce the deformation of the dual steel sheet.

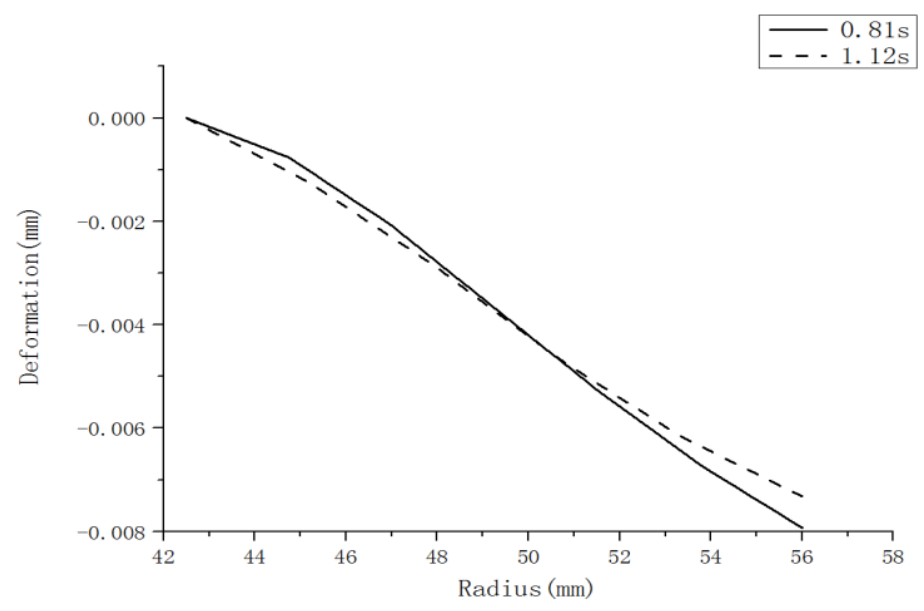

Figure 12.distribution of axial deformation of dual steel sheets along radius

In conclusion, the engagement time has great influence on the temperature field, stress field and axial deformation. Longer engagement time, ride comfort and comfort will be improved, reduce the deformation of the steel sheet. But it can be seen from Figure 6, Figure 9 and Figure 12 that the engagement time is too long, which will result in the increase of temperature, stress, and the increase of temperature gradient and stress gradient, which will affect the service life. But a short engagement time, will cause greater impact, and will have a larger deformation.

\section{Conclusions}

(1) The results show that the engagement time has great influence on the temperature field, the stress field distribution and the axial deformation in the case of constant friction coefficient.

(2)The short engagement time, can affect the ride comfort of the engagement, resulting in a greater impact force, the edge of the dual steel sheet will produce a wave shaped stress distribution, easy to produce curl, the deformation of a single engagement deformation. When the engagement time is longer, the ride comfort is better, but it can lead to higher temperature and higher stress, and the increase of temperature gradient and stress gradient. Therefore, in the design of the clutch, not only 
to consider the ride comfort, but also should consider the engagement time to extend the clutch temperature field, the stress field generated adverse effects.

The results related to the domestic and foreign research results show that the consistent of wet multi disc clutch friction analysis has the high accuracy, the result has certain guiding significance on the design and optimization of the wet clutch.

\section{Acknowledgement}

This project is supported by National Natural Science Foundation of China(NO. 51475402 and 51575166) and Key Program of Science Research of Hunan Provincial Department of Education(NO. 15A185).

\section{Reference}

1. Yang Yalian, Zhang Ka, Qin Datong. Research on Thermal Mechanical Coupling Temperature Field and Stress Field of Multiplate Wet Clutch Steel Disc. CHINA MECH ENG, 20:2740-2744+2781(2014).

2. Xiang Xiaoning, John M K. A Simplified Close Form Approach, for Slipping Clutch Thermal Model. SAE PAPER , 2001 -01-1148 (2001).

3. L. Afferrante, M. Ciavarella, P. Decuzzi, G. Demelio. Transient Analysis of Frictionally Excited Thermoelastic Instability in Multi-disk Clutches and Brakes[J]. Wear ,254 (1/2):136-146(2003).

4. Zhao Shuangmei, Hilmas Gregory E, Dharani Lokeswarappa R. Behavior of a Composite Multidisk Clutch Subjected to Mechanical and Frictionally Excited Thermal Load. Wear , 264 (11/12):1059-1068(2008).

5. Jia Yunhai, Zhang Wenming. Rerearch on Friction Disk Surface Oil Groove Configuration and Temperature Raise in Wet Friction. CHINA J HIGH TRANS, 20(5):112-116(2003).

6. Wang Hongwei, Zhang Xinqin, Zhang Jinle, MA Biao. Simulation of Thermal Load Wet Clutch. J B INST TECHNO, 33(1):47-51(2013).

7. Mansouri M, Holgerson M, Khonsari M M, et al. Thermal and Dynamic Characterization of Wet Clutch Engagement With Provision for Drive Torque. J TRIB, 123(2):313-323(2001).

8. Sun Dongye, Hu Fengbin, Deng Tao, Luo Yong. Simulation and Experiment Warp Characteristic of Wet Multiple Disc Clutch. J CHONGQING UNIV, 33(5):1-6(2010)

9. Zagrodzki P, Truncone S A. Generation of Hot Spots in a Wet Multidisk Clutch During Short-term Engagement. Wear, 254(5-6):474-491(2003).

10. Zhang Jinle, Ma Biao, Zhang Yingfeng, LI Heyan. Study on the Factors Affecting Temperature Field and Stress Field of Wet Shift Clutch. J B INST TECHNO, 06:660-664(2010). 